\title{
Real Killing spinors in neutral signature
}

\section{J. Gutowski ${ }^{a}$ and W.A. Sabra ${ }^{b}$}

${ }^{a}$ Department of Mathematics, University of Surrey, Guildford, GU2 7XH, U.K.

${ }^{b}$ Physics Department, American University of Beirut, Beirut, Lebanon

E-mail: j.gutowski@surrey.ac.uk, ws00@aub.edu.lb

ABSTRACT: Spinorial geometry methods are used to classify solutions admitting Majorana Killing spinors of the minimal 4-dimensional supergravity in neutral signature, with vanishing cosmological constant and a single Maxwell field strength. Two classes of solutions preserving the minimal amount of supersymmetry are found. The first class admits a nullKähler structure and corresponds to a class of self-dual solutions found by Bryant. The second class admits a null and rotation-free geodesic congruence with respect to which a parallel frame can be chosen. Examples of solutions in the former class are pseudo-hyperKähler manifolds; and examples in the latter class include self-dual solutions, as well as a neutral-signature IWP-type solution.

KEYwords: Black Holes in String Theory, Supergravity Models

ARXIV EPRINT: 1905.01910 


\section{Contents}

1 Introduction 1

2 Majorana spinor orbits 3

3 Analysis of the Killing spinor equation 3

3.1 Solutions with Killing spinor $\epsilon=1+e_{12}$

3.1.1 Example: pseudo-Hyper-Kähler metrics 4

3.2 Solutions with Killing spinor $\epsilon=1+e_{12}+e_{1}+e_{2}$

$\begin{array}{ll}3.2 .1 & \text { Example: solutions with } F=0\end{array}$

$\begin{array}{lll}3.2 .2 & \text { Example: solutions with self-dual } F & 12\end{array}$

$\begin{array}{ll}\text { 3.2.3 Example: a neutral signature IWP solution } & 14\end{array}$

4 An alternative Killing spinor equation $\quad 16$

$\begin{array}{lll}5 & \text { Conclusions } & 17\end{array}$

$\begin{array}{lr}\text { A Conventions } & 18\end{array}$

$\begin{array}{lr}\text { B Analysis of condition (3.40) } & 20\end{array}$

$\begin{array}{ll}\text { C Analysis of condition (3.39) } & 21\end{array}$

\section{Introduction}

Much is known about supersymmetric solutions of 4-dimensional supergravity. The classification programme was initiated in work of $[1,2]$ for supergravity theories with Lorentzian signature. The first classification of solutions in a 5-dimensional theory was constructed in [3], by making use of Fierz identity/G-structure analysis. Further extensions of the 4dimensional Lorentzian analysis, using similar methods, was then done in [4-6]. Classifications of solutions with minimal supersymmetry in $\mathrm{D}=11$ supergravity were also found $[7,8]$. Other work on the classification of supersymmetric solutions including more general couplings to hypermultiplets in 4 and 5 dimensions $[9,10]$ and in 6 dimensions using spinorial geometry [11-13] and Fierz identity/G-structure methods [14, 15]. Spinorial geometry techniques have proven to be particularly powerful for the analysis of supersymmetric solutions. This method exploits the fact that spinors can be written as differential forms [16, 17]. This is then applied to classifying supergravity solutions by employing gauge transformations in order to express the Killing spinors in simplified canonical forms, which are then used to 
solve the Killing spinor equations. Such techniques were first used to classify supersymmetric solutions in $\mathrm{D}=11$ supergravity [18], and have also been applied to heterotic and type II supergravity theories [19-22]; see also the review [24] for a comprehensive description of the applications of spinorial geometry to the classification programme.

This work was performed for Lorentzian signature supergravity theories. Analogous classifications have also been performed for Euclidean signature theories in $\mathrm{D}=4$, [25-27], making use of 2-component spinor and spinorial geometry techniques. Einstein-Weyl structures, and the $\mathrm{SU}(\infty)$ Toda equation are among some of the geometric structures associated with such supersymmetric solutions. In contrast, the case of $4 \mathrm{D}$ supersymmetric solutions in neutral signature $(+,+,-,-)$ supergravity theories has received relatively little attention. The analysis of parallel spinors in such theories has been performed in [28-30], and null-Kähler structures are obtained. A further classification of solutions in U(1) gauged neutral signature $4 \mathrm{D}$ supergravity, with a nonzero cosmological constant, and coupled to a single Maxwell field strength, was constructed in [31].

In this paper, we determine, using spinorial geometry techniques, the classification of supersymmetric solutions of the the minimal $\mathrm{D}=4$ neutral signature supergravity, coupled to a Maxwell field strength and with vanishing cosmological constant. The $\mathrm{D}=4$ ungauged neutral signature $\mathcal{N}=2$ supergravity theory, coupled to an arbitrary number of abelian vector multiplets, with scalars taking values in a projective special para-Kähler manifold, was obtained from $\mathrm{D}=11 \mathrm{M}^{*}$ theory [32] via a reduction on $C Y_{3} \times S^{1}$ [33]. In our work, we consider the truncation of this theory to a single Maxwell field strength, with constant scalars. The case of minimal $N=1$ supersymmetric solutions is considered, for which the Killing spinors are Majorana. It is shown that there are two orbits associated with such spinors, and the simplified canonical forms are obtained using appropriately chosen $\operatorname{Spin}(2,2)$ gauge transformations. For both orbits, the necessary and sufficient conditions for a solution to be supersymmetric are determined. One orbit, for which the spinor is chiral, corresponds to a sub-class of the solutions considered in [28-30] for which the spinor is parallel with respect to the Levi-Civita connection and the geometry admits a self-dual instanton. However, the geometry associated with the other orbit, for which the spinor is non-chiral, is novel. Moreover, it does not arise as a limit of solutions constructed in the analysis of [31] for the case of a positive cosmological constant.

The plan of this paper is as follows. In section 2, the canonical Majorana orbits are determined. In section 3, the Killing spinor equations for spinors in the two canonical orbits are analyzed, and the conditions on the geometry and the Maxwell field strength determined. Examples of solutions are also constructed. In section 4, a "Wick rotated" Killing spinor equation is considered, and it is shown that solutions of this Killing spinor equation are in 1-1 correspondence with those considered in section 3 . In section 5, we present our conclusions, and discuss the relationship between the non-chiral spinor orbit geometry, and the classification of [31]. In appendix A the spinorial geometry conventions are presented, and in appendices B and $\mathrm{C}$ some further details of the analysis of the Killing spinor equation conditions in section 3 are given. 


\section{Majorana spinor orbits}

The Killing spinor equation (KSE) which we consider is given by

$$
D_{\mu} \epsilon \equiv \nabla_{\mu} \epsilon-\frac{1}{4} \not F \Gamma_{\mu} \epsilon=0
$$

where $F$ is the Maxwell field strength, which satisfies

$$
d F=0, \quad d \star F=0 .
$$

In particular, if $\epsilon$ satisfies (2.1) then so does $C * \epsilon$. Hence it is sufficient to consider Majorana spinors $\epsilon$ which satisfy $C * \epsilon=\epsilon$. We begin by choosing simple canonical forms for the Majorana spinors. A basis (over $\mathbb{R}$ ) for Majorana spinors $\left\{\eta_{1}, \eta_{2}, \eta_{3}, \eta_{4}\right\}$, satisfying $C * \eta_{i}=\eta_{i}$, is given by

$$
\eta_{1}=1+e_{12}, \quad \eta_{2}=i\left(1-e_{12}\right), \quad \eta_{3}=e_{1}+e_{2}, \quad \eta_{4}=i\left(e_{1}-e_{2}\right) .
$$

Consider the spaces of Majorana spinors $\operatorname{Span}_{\mathbb{R}}\left(\left\{\eta_{1}, \eta_{2}\right\}\right)$ and $\operatorname{Span}_{\mathbb{R}}\left(\left\{\eta_{3}, \eta_{4}\right\}\right)$. We remark that the spinor orbits for the case of $(2,2)$ signature presented in [28] correspond to Majorana spinors, where the spinors are taken to be in $\mathbb{R}^{2} \oplus \mathbb{R}^{2}$. The two copies of $\mathbb{R}^{2}$ are identified with $\operatorname{Span}_{\mathbb{R}}\left(\left\{\eta_{1}, \eta_{2}\right\}\right)$ and $\operatorname{Span}_{\mathbb{R}}\left(\left\{\eta_{3}, \eta_{4}\right\}\right)$. To evaluate canonical forms for Majorana spinors, we use only real $\operatorname{Spin}(2,2)$ gauge transformations which commute with $C *$ and hence preserve the Majorana condition. Here we use the conventions presented in appendix A.

The action of $\sigma_{1}, \sigma_{2}, i \sigma_{3}$ associated with (real) $\operatorname{Spin}(2,2)$ gauge transformations, acting independently on these vector spaces is generated by

$$
T_{1}=\left(\begin{array}{cc}
1 & 0 \\
0 & -1
\end{array}\right), \quad T_{2}=\left(\begin{array}{cc}
0 & -1 \\
-1 & 0
\end{array}\right), \quad T_{3}=\left(\begin{array}{cc}
0 & -1 \\
1 & 0
\end{array}\right) .
$$

It follows that a $\mathrm{SL}(2, \mathbb{R})$ transformation generated by $T_{1}, T_{2}, T_{3}$ can be used to write $\epsilon_{1} \in \operatorname{Span}_{\mathbb{R}}\left(\left\{\eta_{1}, \eta_{2}\right\}\right)$ as $\epsilon_{1}=a . \eta_{1}$ where $a=0$ or $a=1$. A similar argument can be used to write $\epsilon_{2} \in \operatorname{Span}_{\mathbb{R}}\left(\left\{\eta_{3}, \eta_{4}\right\}\right)$ as $\epsilon_{2}=b . \eta_{3}$ for $b=0$ or $b=1$.

So there are three possible canonical Majorana spinors corresponding to

$$
\epsilon=\eta_{1}, \quad \text { or } \quad \epsilon=\eta_{3}, \quad \text { or } \quad \epsilon=\eta_{1}+\eta_{3}
$$

however $\eta_{1}$ and $\eta_{3}$ are also related by the Pin $(2,2)$ transformation generated by $\gamma_{1}$, so there are two canonical Majorana spinors given by

$$
\epsilon=1+e_{12}, \quad \text { or } \quad \epsilon=1+e_{12}+e_{1}+e_{2} .
$$

We next analyse the conditions obtained from (2.1) in these two cases.

\section{Analysis of the Killing spinor equation}

In this section, we analyse the Killing spinor equation (2.1). For each of the two classes of canonical Majorana spinors given in (2.6) we derive the necessary and sufficient conditions on the geometry and the Maxwell field strength. 


\subsection{Solutions with Killing spinor $\epsilon=1+e_{12}$}

Consider first the KSE (2.1) in the case for which $\epsilon=1+e_{12}$. We find, on acting with $\gamma_{5}$ on (2.1), the conditions

$$
\nabla_{\mu} \epsilon=0, \quad \not F \Gamma_{\mu} \epsilon=0
$$

As the Majorana spinor $1+e_{12}$ is parallel, the geometry corresponds to one found in [28]. The condition $\not F \Gamma_{\mu}\left(1+e_{12}\right)=0$ is also equivalent to $F=\star F$, where $\epsilon_{1 \overline{1} 2 \overline{2}}=1$.

The bilinear given by

$$
\chi_{\mu \nu}=i \mathcal{B}\left(\epsilon, \Gamma_{5} \Gamma_{\mu \nu} \epsilon\right)
$$

is represented by the two-form

$$
\chi=2\left(\mathbf{e}^{1} \wedge \mathbf{e}^{2}+\mathbf{e}^{\overline{1}} \wedge \mathbf{e}^{\overline{2}}\right)+2 i\left(\mathbf{e}^{1} \wedge \mathbf{e}^{\overline{1}}-\mathbf{e}^{2} \wedge \mathbf{e}^{\overline{2}}\right) .
$$

Using the KSE, it can be shown that

$$
\nabla \chi=0
$$

Moreover $\chi$ is null $\left(\chi^{2}=0\right)$. Therefore the solutions admit a null-Kähler structure. The metric can be written in the form [28-30]

$$
d s^{2}=d w d x+d z d y-S_{x^{2}} d z^{2}-S_{y^{2}} d w^{2}+2 S_{x y} d w d z
$$

where we have used the notation

$$
S_{x^{2}}=\frac{\partial^{2} S}{\partial x^{2}}, \quad S_{y^{2}}=\frac{\partial^{2} S}{\partial y^{2}}, \quad S_{x y}=\frac{\partial^{2} S}{\partial x \partial y} .
$$

The vanishing of the Ricci curvature implies the conditions:

$$
\begin{array}{r}
S_{x^{2} y w}+S_{x y^{2} z}-S_{x^{2} y} S_{x y^{2}}+S_{x^{3}} S_{y^{3}}+S_{y^{2}} S_{x^{3} y}-2 S_{x y} S_{x^{2} y^{2}}+S_{x^{2}} S_{x y^{3}}=0 \\
-S_{x^{3} w}-S_{x^{2} y z}+2\left(S_{x^{2} y}\right)^{2}-2 S_{x^{3}} S_{x y^{2}}-S_{y^{2}} S_{x^{4}}+2 S_{x y} S_{x^{3} y}-S_{x^{2}} S_{x^{2} y^{2}}=0 \\
S_{x y^{2} w}+S_{y^{3} z}-2\left(S_{x y^{2}}\right)^{2}+2 S_{x^{2} y} S_{y^{3}}+S_{y^{2}} S_{x^{2} y^{2}}-2 S_{x y} S_{x y^{3}}+S_{x^{2}} S_{y^{4}}=0 .
\end{array}
$$

We remark that such solutions always admit a self-dual covariantly constant Maxwell field strength, given by $F=k \chi$ for constant $k$.

\subsubsection{Example: pseudo-Hyper-Kähler metrics}

Further conditions can be obtained if one assumes extended supersymmetry. For example, consider a $N=2$ solution which, in addition to the Killing spinor $\epsilon=\left(1+e_{12}\right)$, also admits a further Killing spinor given by $\eta=i\left(1-e_{12}\right)$.

The two form spinor bilinear given by

$$
\left(\chi_{2}\right)_{\mu \nu}=i \mathcal{B}\left(\eta, \Gamma_{5} \Gamma_{\mu \nu} \eta\right)
$$


is represented by

$$
\chi_{2}=2 i\left(\mathbf{e}^{1} \wedge \mathbf{e}^{\overline{1}}-\mathbf{e}^{2} \wedge \mathbf{e}^{\overline{2}}\right)-2\left(\mathbf{e}^{1} \wedge \mathbf{e}^{2}+\mathbf{e}^{\overline{1}} \wedge \mathbf{e}^{\overline{2}}\right) .
$$

Moreover we calculate a third 2-form spinor bilinear given by

$$
\left(\chi_{3}\right)_{\mu \nu}=i \mathcal{B}\left(\epsilon, \Gamma_{5} \Gamma_{\mu \nu} \eta\right) \text {. }
$$

This is given by

$$
\chi_{3}=-2 i\left(\mathbf{e}^{1} \wedge \mathbf{e}^{2}-\mathbf{e}^{\overline{1}} \wedge \mathbf{e}^{\overline{2}}\right) .
$$

Therefore we have the three bilinears:

$$
\begin{aligned}
& \chi_{1}=2\left(\mathbf{e}^{1} \wedge \mathbf{e}^{2}+\mathbf{e}^{\overline{1}} \wedge \mathbf{e}^{\overline{2}}\right)+2 i\left(\mathbf{e}^{1} \wedge \mathbf{e}^{\overline{1}}-\mathbf{e}^{2} \wedge \mathbf{e}^{\overline{2}}\right) \\
& \chi_{2}=2 i\left(\mathbf{e}^{1} \wedge \mathbf{e}^{\overline{1}}-\mathbf{e}^{2} \wedge \mathbf{e}^{\overline{2}}\right)-2\left(\mathbf{e}^{1} \wedge \mathbf{e}^{2}+\mathbf{e}^{\overline{1}} \wedge \mathbf{e}^{\overline{2}}\right) \\
& \chi_{3}=-2 i\left(\mathbf{e}^{1} \wedge \mathbf{e}^{2}-\mathbf{e}^{\overline{1}} \wedge \mathbf{e}^{\overline{2}}\right) .
\end{aligned}
$$

We write

$$
\begin{aligned}
& J_{1}=\frac{1}{4}\left(\chi_{1}-\chi_{2}\right)=\left(\mathbf{e}^{1} \wedge \mathbf{e}^{2}+\mathbf{e}^{\overline{1}} \wedge \mathbf{e}^{\overline{2}}\right) \\
& J_{2}=\frac{1}{4}\left(\chi_{1}+\chi_{2}\right)=i\left(\mathbf{e}^{1} \wedge \mathbf{e}^{\overline{1}}-\mathbf{e}^{2} \wedge \mathbf{e}^{\overline{2}}\right) \\
& J_{3}=\frac{1}{2} \chi_{3}=-i\left(\mathbf{e}^{1} \wedge \mathbf{e}^{2}-\mathbf{e}^{\overline{1}} \wedge \mathbf{e}^{\overline{2}}\right) .
\end{aligned}
$$

Then the KSE imply that

$$
\nabla J_{1}=\nabla J_{2}=\nabla J_{3}=0
$$

with

$$
J_{1}^{2}=-J_{2}^{2}=J_{3}^{2}=1
$$

and

$$
J_{1} J_{2}=-J_{2} J_{1}=J_{3}, \quad J_{1} J_{3}=-J_{3} J_{1}=J_{2}, \quad J_{2} J_{3}=-J_{3} J_{2}=J_{1} .
$$

Hence, the geometry admits a pseudo-hyper-Kähler metric. The additional spinor imposes the following integrability condition on the Riemann curvature tensor of the metric (3.5)

$$
R_{\mu \nu \alpha \beta} \Gamma^{\alpha \beta} 1=0 .
$$

Hence, the Riemann curvature is anti-self-dual, in contrast to the null Kähler form $d z \wedge d w$ which is self-dual with respect to the volume form dvol $=\frac{1}{4} d x \wedge d y \wedge d w \wedge d z$. The antiself-duality of the curvature imposes a further condition on the function $S$ in addition to those imposed by Ricci flatness given in (3.7), which is:

$$
\begin{aligned}
& 2 S_{x y z w}+S_{x^{2} w^{2}}+S_{z^{2} y^{2}}+S_{x^{2} y} S_{y^{2} z}+S_{x y^{2}} S_{x^{2} w}+S_{x^{3}} S_{y^{2} w}+S_{y^{3}} S_{x^{2} z} \\
&-2 S_{x^{2} y} S_{x y w}-2 S_{x y^{2}} S_{x y z} S_{y^{2}} S_{x^{3} w}+S_{y^{2}} S_{x^{2} y z}-2 S_{x y} S_{x^{2} y w} \\
&-2 S_{x y} S_{x y^{2} z}+S_{x^{2}} S_{x y^{2} w}+S_{x^{2}} S_{y^{3} z}=0 .
\end{aligned}
$$


3.2 Solutions with Killing spinor $\epsilon=1+e_{12}+e_{1}+e_{2}$

Next, consider the case for which the Killing spinor is $\epsilon=1+e_{12}+e_{1}+e_{2}$. Then the linear system obtained from (2.1) is as follows:

$$
\begin{aligned}
-\omega_{1,1 \overline{1}}+\omega_{1,2 \overline{2}}+2 i \omega_{1, \overline{1} \overline{2}} & =\sqrt{2}\left(-F_{1 \overline{1}}+F_{2 \overline{2}}\right) \\
\omega_{1,1 \overline{1}}-\omega_{1,2 \overline{2}}-2 i \omega_{1,12} & =-2 \sqrt{2} i F_{12} \\
\omega_{1,1 \overline{1}}+\omega_{1,2 \overline{2}}-2 i \omega_{1,1 \overline{2}} & =-2 \sqrt{2} i F_{1 \overline{2}} \\
-\omega_{1,1 \overline{1}}-\omega_{1,2 \overline{2}}+2 i \omega_{1, \overline{1} 2} & =-\sqrt{2}\left(F_{1 \overline{1}}+F_{2 \overline{2}}\right) \\
-\omega_{2,1 \overline{1}}+\omega_{2,2 \overline{2}}+2 i \omega_{2, \overline{1} \overline{2}} & =\sqrt{2} i\left(-F_{1 \overline{1}}+F_{2 \overline{2}}\right) \\
\omega_{2,1 \overline{1}}-\omega_{2,2 \overline{2}}-2 i \omega_{2,12} & =2 \sqrt{2} F_{12} \\
\omega_{2,1 \overline{1}}+\omega_{2,2 \overline{2}}-2 i \omega_{2,1 \overline{2}} & =-\sqrt{2} i\left(F_{1 \overline{1}}+F_{2 \overline{2}}\right) \\
-\omega_{2,1 \overline{1}}-\omega_{2,2 \overline{2}}+2 i \omega_{2, \overline{1} 2} & =2 \sqrt{2} F_{\overline{1} 2} .
\end{aligned}
$$

To proceed, consider the 1-form $W$ defined by

$$
W_{\mu}=i \mathcal{B}\left(\epsilon, \Gamma_{5} \Gamma_{\mu} \epsilon\right)
$$

and the 2 -form $\chi$ given by

$$
\chi_{\mu \nu}=i \mathcal{B}\left(\epsilon, \Gamma_{5} \Gamma_{\mu \nu} \epsilon\right) .
$$

These spinor bilinears are given explicitly by

$$
W=2 \sqrt{2} i\left(\mathbf{e}^{1}-\mathbf{e}^{\overline{1}}\right)-2 \sqrt{2}\left(\mathbf{e}^{2}+\mathbf{e}^{\overline{2}}\right), \quad \chi=W \wedge \theta
$$

where

$$
\theta=\frac{1}{\sqrt{2}}\left(\mathbf{e}^{1}+\mathbf{e}^{\overline{1}}\right) .
$$

Then

$$
\nabla_{\nu} W_{\mu}=\frac{1}{2} \eta_{\mu \nu} F_{\lambda_{1} \lambda_{2}} \chi^{\lambda_{1} \lambda_{2}}+F_{\nu \lambda} \chi_{\mu}^{\lambda}+F_{\mu \lambda} \chi^{\lambda}{ }_{\nu}
$$

and

$$
\nabla_{\sigma} \chi_{\mu \nu}=F_{\sigma \mu} W_{\nu}-F_{\sigma \nu} W_{\mu}-F_{\mu \nu} W_{\sigma}+\eta_{\sigma \mu}\left(i_{W} F\right)_{\nu}-\eta_{\sigma \nu}\left(i_{W} F\right)_{\mu}
$$

We remark that the conditions (3.22), (3.23), (3.24), (3.25) are equivalent to the linear system (3.19). To proceed with the analysis of these conditions, note that (3.24) implies that

$$
d W=0, \quad \nabla^{\mu} W_{\mu}=0 .
$$

Furthermore, we also have as a consequence of (3.25)

$$
W \wedge(F+d \theta)=0
$$


and also

$$
\left(i_{W} F\right)_{\mu}=\nabla^{\lambda} \chi_{\lambda \mu}
$$

On substituting (3.27) and (3.28) into (3.25), it follows that (3.25) is equivalent to

$$
W_{\sigma} F_{\mu \nu}=-\frac{1}{2} \nabla_{\sigma} \chi_{\mu \nu}-\frac{1}{2}(W \wedge d \theta)_{\sigma \mu \nu}+\frac{1}{2} \eta_{\sigma \mu} \nabla^{\lambda} \chi_{\lambda \nu}-\frac{1}{2} \eta_{\sigma \nu} \nabla^{\lambda} \chi_{\lambda \mu} .
$$

This condition determines all components of $F$ in terms of the geometry. On using (3.29) to eliminate $F$ from (3.24), it follows that (3.24) is equivalent to

$$
\nabla_{W} \theta=\left(\nabla^{\nu} \theta_{\nu}\right) W
$$

To proceed, consider (3.27). This implies that

$$
F=-d \theta+W \wedge \psi
$$

for some 1-form $\psi$. The Bianchi identity implies that

$$
W \wedge d \psi=0
$$

and hence here exists a function $\mathcal{G}$ such that

$$
F=-d \theta+W \wedge d \mathcal{G}=-d(\theta+\mathcal{G} W)
$$

There is a freedom to make the redefinition

$$
\hat{\theta}=\theta+\mathcal{G} W
$$

and we note that $\hat{\theta}^{2}=1$ and $\hat{\theta}, W$ are orthogonal. On making this redefinition, and dropping the $\hat{,}$ we take without loss of generality

$$
F=-d \theta .
$$

In addition, on making use of (3.30), it follows that

$$
\nabla^{\lambda} \chi_{\lambda \mu}=-\left(i_{W} d \theta\right)_{\mu}+\left(\nabla^{\lambda} \theta_{\lambda}\right) W_{\mu}
$$

and so (3.28) implies

$$
\nabla^{\mu} \theta_{\mu}=0
$$

hence (3.30) simplifies to

$$
\nabla_{W} \theta=0
$$

It remains to evaluate (3.24) and also (3.29). On setting $F=-d \theta,(3.24)$ is equivalent to

$$
\nabla_{\nu} W_{\mu}=\eta_{\mu \nu} \theta^{\lambda} W^{\rho}(d \theta)_{\lambda \rho}+2\left(i_{W} d \theta\right)_{(\nu} \theta_{\mu)}-2\left(i_{\theta} d \theta\right)_{(\nu} W_{\mu)}
$$


and (3.29) is equivalent to

$$
\begin{aligned}
\frac{1}{2} W_{\sigma}(d \theta)_{\mu \nu}-W_{[\mu} \nabla_{\nu]} \theta_{\sigma}-\left(-\left(i_{\theta} d \theta\right)_{\sigma} W_{[\mu}+\theta_{\sigma}\left(i_{W} d \theta\right)_{[\mu}-W_{\sigma}\left(i_{\theta} d \theta\right)_{[\mu}\right) \theta_{\nu]} & \\
-\eta_{\sigma[\mu}\left(\theta_{\nu]}(d \theta)_{\lambda \rho} \theta^{\lambda} W^{\rho}+\left(i_{W} d \theta\right)_{\nu]}\right) & =0 .
\end{aligned}
$$

The conditions on $\nabla \theta$ obtained from (3.40) can be simplified, making use of (3.37) and (3.38) to give

$$
\star(\theta \wedge d \theta)=\nabla_{\tau} \theta
$$

where $\tau$ is orthogonal to $\theta$ and $W$, and satisfies $\tau^{2}=-1$. Details of this analysis are given in appendix B.

The condition (3.39) is equivalent, together with (3.26), to

$$
\nabla_{\tau} W=\star(W \wedge d \theta)
$$

and

$$
\nabla_{V} W=\star(\tau \wedge d \theta)-i_{\theta} d \theta
$$

where, with respect to the frame $\{V, W, \tau, \theta\}$, the metric is

$$
d s^{2}=2 V W+\theta^{2}-\tau^{2}
$$

with volume form dvol $=W \wedge V \wedge \tau \wedge \theta$. Details of this analysis are given in appendix C.

Hence, the geometric conditions obtained so far, associated with the frame (3.44), can be written as

$$
d \star \theta=0, \quad \nabla_{W} \theta=0, \quad \nabla_{\tau} \theta=\star(\theta \wedge d \theta)
$$

and

$$
d W=0, \quad d \star W=0, \quad \nabla_{\tau} W=\star(W \wedge d \theta), \quad \nabla_{V} W=\star(\tau \wedge d \theta)-i_{\theta} d \theta
$$

and the gauge field is

$$
F=-d \theta
$$

We remark that these conditions, as well as the metric (3.44) are invariant under the redefinitions

$$
V^{\prime}=V-\beta \tau+\frac{1}{2} \beta^{2} W, \quad \tau^{\prime}=\tau-\beta W .
$$

Next we consider the gauge field equations. In order to analyse these, we note that

$$
\star \chi=-W \wedge \tau
$$

and that the condition (3.25) can be rewritten as

$$
\nabla_{\sigma} \star \chi_{\mu \nu}=-W_{\sigma} \star F_{\mu \nu}+W_{\mu} \star F_{\nu \sigma}-W_{\nu} \star F_{\mu \sigma}+\eta_{\sigma \mu}\left(i_{W} \star F\right)_{\nu}-\eta_{\sigma \nu}\left(i_{W} \star F\right)_{\mu}
$$


from which it follows that

$$
d \star \chi=W \wedge \star F
$$

and therefore

$$
W \wedge(\star F-d \tau)=0 .
$$

Hence, it follows that there exists a 1-form $\psi$ such that

$$
\star F=d \tau+W \wedge \psi
$$

The gauge field equations $d \star F=0$ then imply that

$$
W \wedge d \psi=0
$$

Hence there exists a function $\mathcal{H}$ such that

$$
\star F=d \tau+W \wedge d \mathcal{H} .
$$

By making use of a redefinition of the form (3.48) we can without loss of generality set $\mathcal{H}=0$, and then (dropping the primes)

$$
\star F=d \tau .
$$

As we have already found the condition $F=-d \theta$, the gauge field equations are equivalent to

$$
\star d \theta=-d \tau .
$$

On substituting (3.57) into the condition (3.42), and making use of the closure of $W$, it follows that (3.42) is equivalent to

$$
\nabla_{W} \tau=0
$$

As we also have the conditions $\nabla_{W} W=0$ and $\nabla_{W} \theta=0$, and the metric must also be parallel with respect to $W$, this also implies that $\nabla_{W} V=0$ as well, i.e. the frame $\{V, W, \tau, \theta\}$ is parallel with respect to $W$. We also remark that the condition (3.43) can be rewritten as

$$
\nabla_{V} W+\nabla_{W} V-\nabla_{\tau} \tau+\nabla_{\theta} \theta=0 .
$$

It remains to consider the Einstein equations. The integrability conditions of the KSE, together with the Bianchi identity and gauge field equations, imply that

$$
E_{\mu \nu} \Gamma^{\mu} \epsilon=0
$$

where $E_{\mu \nu}=0$ is equivalent to the Einstein equations. Then (3.60) implies that

$$
E_{\mu \nu} W^{\nu}=0 \text {. }
$$


Furthermore, (3.60) implies that

$$
\left(E_{\mu \rho}+\Gamma_{\rho}^{\nu} E_{\mu \nu}\right) \epsilon=0 .
$$

On making use of the condition $\mathcal{B}\left(\epsilon, \Gamma_{5} \epsilon\right)=0$ and $\mathcal{B}(\epsilon, \epsilon)=0$, the above expression implies that

$$
\chi_{\rho}^{\nu} E_{\mu \nu}=0, \quad \text { and } \quad \star \chi_{\rho}{ }^{\nu} E_{\mu \nu}=0
$$

or equivalently

$$
E_{\mu \nu} \theta^{\nu}=0, \quad E_{\mu \nu} \tau^{\nu}=0 .
$$

Then (3.61) and (3.64) imply that the only component of the Einstein equations not implied to hold by supersymmetry is that corresponding to

$$
V^{\mu} V^{\nu} E_{\mu \nu}=0 \text {. }
$$

To summarize; in the case for which the Killing spinor is $\epsilon=1+e_{12}+e_{1}+e_{2}$, the necessary and sufficient conditions for supersymmetry are:

$$
d \star \theta=0, \quad \nabla_{\tau} \theta=i_{\theta}(d \tau), \quad \star d \theta=-d \tau
$$

and

$$
d W=0, \quad \nabla_{W} \mathbf{e}^{a}=0, \quad \nabla_{V} W+\nabla_{W} V-\nabla_{\tau} \tau+\nabla_{\theta} \theta=0
$$

where $\mathbf{e}^{a}$ denotes the frame $\{V, W, \tau, \theta\}$, with respect to which the metric is

$$
d s^{2}=2 V W+\theta^{2}-\tau^{2}
$$

and the Maxwell field strength is

$$
F=-d \theta
$$

The conditions (3.66) and (3.67) imply that $W$ is co-closed, so this condition has been omitted from (3.66), (3.67). These conditions are also sufficient to ensure that all components of the Bianchi identity and gauge field equations hold, and that all but one component of the Einstein equations are satisfied. The remaining component of the Einstein equations which is not implied by supersymmetry is (3.65), which must be imposed as an additional condition to the above.

\subsubsection{Example: solutions with $F=0$}

For solutions with $F=0$, we note that if $\epsilon$ is a parallel Killing spinor, then so is $\gamma_{5} \epsilon$. So, taking $\epsilon=1+e_{12}+e_{1}+e_{2}$, this implies that both $1+e_{12}$ and $e_{1}+e_{2}$ are independently parallel, i.e. such solutions are actually $N=2$ solutions which admit two spinors $\epsilon_{1}=1+e_{12}$ 
and $\epsilon_{2}=e_{1}+e_{2}$. These are therefore special cases of the solutions considered in section 3.1, and have also been considered in [28].

It is however instructive to consider how such solutions appear in terms of the classification of solutions constructed for the spinor $\epsilon=1+e_{12}+e_{1}+e_{2}$. In particular, the condition $F=0$ together with (3.47) and (3.57) implies that

$$
d \theta=d \tau=0
$$

and hence local co-ordinates $v, u, x, y$ can be introduced such that

$$
W=d u, \quad V=d v+A d u+h_{1} d x+h_{2} d y, \quad \theta=d x, \quad \tau=d y
$$

with the vector field dual to $W$ given by $W=\frac{\partial}{\partial v}$. Then

$$
\mathcal{L}_{W} W=\mathcal{L}_{W} \tau=\mathcal{L}_{W} \theta=0
$$

because $W, \tau$ and $\theta$ are closed. Furthermore, the conditions

$$
\nabla_{V} W=\nabla_{W} V=0
$$

imply that

$$
\mathcal{L}_{W} V=0
$$

also, so $W$ is an isometry and therefore parallel. In particular, this implies that the functions $A, h_{1}, h_{2}$ are independent of $v$. A co-ordinate change of the form $v=v^{\prime}+$ $f_{1}(u, x, y)$ can then be used to set, without loss of generality $h_{2}=0$, so $V=d v+A d u+h d x$ wheren $A$ and $h$ are independent of $v$ (dropping the prime)

Next, the condition $\nabla_{\tau} \theta=0$ implies that $\partial_{y} h=0$, so a further co-ordinate transformation of the form $v=v^{\prime}+f_{2}(u, x)$ can be used to set $h=0$ as well. It follows that in these co-ordinates, the metric is

$$
d s^{2}=2 d u(d v+A d u)+d x^{2}-d y^{2} .
$$

It remains to impose the Einstein equations, which are equivalent to $R_{v v}=0$. This implies that

$$
\frac{\partial^{2} A}{\partial y^{2}}-\frac{\partial^{2} A}{\partial x^{2}}=0
$$

Hence, $A$ is a $u$-dependent function which is harmonic on $\mathbb{R}^{1,1}$, i.e.

$$
A=A_{+}(u, x+y)+A_{-}(u, x-y) .
$$

In particular, the Riemann curvature tensor of the metric (3.75) is self-dual if and only if

$$
\frac{\partial^{2} A_{+}}{\partial(x+y)^{2}}=0
$$


and the Riemann curvature tensor is anti-self-dual if and only if

$$
\frac{\partial^{2} A_{-}}{\partial(x-y)^{2}}=0
$$

Such solutions have already been constructed in [34], albeit in different co-ordinates. In particular, the metric is written in terms of co-ordinates $\{p, t, u, v\}$ as

$$
d s^{2}=d p d t-\frac{1}{2} p^{2} d u d v-\frac{1}{2} p^{2} H d u^{2}
$$

where $H=H(p, u)$. On changing co-ordinates to $\{p, \hat{t}, \hat{u}, \hat{v}\}$, where

$$
\hat{u}=p u, \quad \hat{v}=p v, \quad \hat{t}=t+\frac{1}{2} p u v
$$

the metric is

$$
d s^{2}=d p d \hat{t}-\frac{1}{2} d \hat{u} d \hat{v}-\frac{1}{2} H(d \hat{u})^{2}+p^{-1} \hat{u} H d \hat{u} d p-\frac{1}{2} p^{-2}(\hat{u})^{2} H d p^{2}
$$

for $H=H(p, \hat{u})$. Finally, on setting

$$
\hat{t}=\tilde{t}+g_{1}(p, \hat{u}), \quad \hat{v}=\tilde{v}+g_{2}(p, \hat{u})
$$

where $g_{1}, g_{2}$ are chosen to satisfy

$$
\frac{\partial g_{1}}{\partial p}=\frac{1}{2} p^{-2}(\hat{u})^{2} H, \quad \frac{1}{2} \frac{\partial g_{2}}{\partial p}=p^{-1} \hat{u} H+\frac{\partial g_{1}}{\partial \hat{u}}
$$

the metric is

$$
d s^{2}=d p d \tilde{t}-\frac{1}{2} d \hat{u} d \tilde{v}+\tilde{H}(d \hat{u})^{2}
$$

where $\tilde{H}=\tilde{H}(p, \hat{u})$ is given by

$$
\tilde{H}=-\frac{1}{2} H-\frac{1}{2} \frac{\partial g_{2}}{\partial \hat{u}} .
$$

The metric (3.85) corresponds to the solution given in (3.75), in the special case for which the wave profile function $A$ contains only either left (or right) moving modes, on making a trivial re-labelling of the co-ordinates.

\subsubsection{Example: solutions with self-dual $F$}

A more general class of solutions is obtained by taking non-zero, but self-dual, $F$. If $F=\star F$, then the geometric conditions imply that

$$
d(\theta+\tau)=0
$$

and also

$$
\nabla_{\theta+\tau} \theta=0 .
$$


We can then introduce local co-ordinates $\{v, u, x, y\}$ so that the vector field $W$ is $W=\frac{\partial}{\partial v}$, and the 1-forms are

$$
\begin{aligned}
W & =d u \\
V & =d v+H d u+h_{1} d x+h_{2} d y \\
\tau+\theta & =d x \\
\tau-\theta & =q d u+p_{1} d x+p_{2} d y .
\end{aligned}
$$

Requiring that $d \star W=0$ implies that $\partial_{v} p_{2}=0$, and hence on defining

$$
\hat{y}=\int p_{2} d y
$$

we can without loss of generality take $p_{2}=1$. The conditions $\nabla_{W} \mathbf{e}^{a}=0$ further imply that

$$
\partial_{v} h_{2}=0
$$

and

$$
\partial_{v}\left(h_{1}+\frac{1}{2} q\right)=0 \text {. }
$$

The condition $\partial_{v} h_{2}=0$ implies that on making a co-ordinate transformation of the form $v=\hat{v}+\mathcal{F}(u, x, y)$, we can without loss of generality drop the hat, and set $h_{2}=0$.

Next, the condition (3.88) implies that

$$
\partial_{y}\left(h_{1}+\frac{1}{2} q\right)=0 .
$$

The conditions (3.92) and (3.93) imply that

$$
h_{1}=-\frac{1}{2} q+\psi(u, x) .
$$

By making a co-ordinate transformation of the form

$$
v=\tilde{v}+\mathcal{G}(u, x)
$$

we can without loss of generality set $\psi=0$, and take $h_{1}=-\frac{1}{2} q$.

After making these simplifications, the metric is

$$
d s^{2}=2 d u\left(d v+H d u-\frac{1}{2} q d x\right)-d x\left(q d u+p_{1} d x+d y\right) .
$$

The remaining geometric conditions are obtained from the condition (3.59), which implies

$$
\partial_{y} q+\partial_{v} H=0
$$

and $d \star \theta=0$ implies

$$
\partial_{y} p_{1}-\frac{1}{2} \partial_{v} q=0
$$


and the condition $d \theta=\star d \theta$ further implies

$$
\partial_{x} q-\partial_{u} p_{1}+\partial_{v}\left(\frac{1}{2} q^{2}+p_{1} H\right)=0
$$

The conditions (3.97), (3.98) and (3.99) have a simple geometric interpretation. In particular, on defining

$$
\omega=d u \wedge d x, \quad \text { and } \quad \hat{\omega}=\left(d v+H d u-\frac{1}{2} q d x\right) \wedge\left(q d u+p_{1} d x+d y\right)
$$

it is straightforward to show that $\omega$ and $\hat{\omega}$ define null almost complex structures with respect to the metric (3.96). The conditions (3.97), (3.98) and (3.99) are then equivalent to

$$
\nabla \omega=0, \quad i_{W} d \hat{\omega}=0 .
$$

In particular, the metric admits a null-Kähler structure with respect to $\omega$. This is to be expected, as the Killing spinor equation (2.1), together with the self-duality condition $F=\star F$, imply that the chiral spinor $e_{1}+e_{2}$ is parallel with respect to the Levi-Civita connection. In contrast, the conditions (3.97), (3.98) and (3.99) are insufficient to imply integrability of the null almost complex structure $\hat{\omega}$.

The conditions (3.97), (3.98), and (3.99) imply that all components of the Ricci tensor vanish, with the exception of $R_{u u}$, where

$$
\begin{aligned}
R_{u u}= & 2 H \partial_{v}^{2} H+4 q \partial_{y} \partial_{v} H+4 \partial_{y} \partial_{x} H+2 \partial_{y} \partial_{u} q+2 \partial_{v} H \partial_{y} q \\
& -2 \partial_{v} q \partial_{y} H-4 \partial_{y} H \partial_{y} p_{1}-2\left(\partial_{y} q\right)^{2}-4 p_{1} \partial_{y}^{2} H .
\end{aligned}
$$

The condition $R_{u u}=0$ must also be imposed.

\subsubsection{Example: a neutral signature IWP solution}

A final example is that of a neutral signature IWP-type solution, which has metric

$$
d s^{2}=\lambda^{2} \sigma^{2}(d t+\phi)^{2}+\frac{1}{\lambda^{2} \sigma^{2}}\left(d x^{2}-d w d \bar{w}\right)
$$

where $t, x$ are real co-ordinates and $w$ is a complex co-ordinate. $\lambda$ and $\sigma$ are functions of the co-ordinates $x, w, \bar{w}$, and $\phi=\phi_{x} d x+\phi_{w} d w+\phi_{\bar{w}} d \bar{w}$ is a real 1-form whose components are $t$-independent. The 1 -form $\phi$ satisfies

$$
\begin{aligned}
d \phi= & \frac{1}{\lambda^{2} \sigma^{2}}\left(-i\left(\sigma^{-1} \partial_{x} \sigma-\lambda^{-1} \partial_{x} \lambda\right) d w \wedge d \bar{w}\right. \\
& -2 i\left(\sigma^{-1} \partial_{w} \sigma-\lambda^{-1} \partial_{w} \lambda\right) d w \wedge d x \\
& \left.+2 i\left(\sigma^{-1} \partial_{\bar{w}} \sigma-\lambda^{-1} \partial_{\bar{w}} \lambda\right) d \bar{w} \wedge d x\right)
\end{aligned}
$$

and the gauge field is

$$
\begin{aligned}
F= & -\frac{1}{2} d\left[\left(\lambda^{2}+\sigma^{2}\right)(d t+\phi)\right]-\frac{i}{4} \partial_{x}\left(\frac{1}{\lambda^{2}}-\frac{1}{\sigma^{2}}\right) d w \wedge d \bar{w} \\
& +\frac{i}{2} \partial_{w}\left(\frac{1}{\lambda^{2}}-\frac{1}{\sigma^{2}}\right) d x \wedge d w-\frac{i}{2} \partial_{\bar{w}}\left(\frac{1}{\lambda^{2}}-\frac{1}{\sigma^{2}}\right) d x \wedge d \bar{w}
\end{aligned}
$$


which has dual

$$
\begin{aligned}
\star F= & -\frac{1}{2} d\left[\left(\lambda^{2}-\sigma^{2}\right)(d t+\phi)\right]+\frac{i}{4} \partial_{x}\left(\frac{1}{\lambda^{2}}+\frac{1}{\sigma^{2}}\right) d w \wedge d \bar{w} \\
& -\frac{i}{2} \partial_{w}\left(\frac{1}{\lambda^{2}}+\frac{1}{\sigma^{2}}\right) d x \wedge d w+\frac{i}{2} \partial_{\bar{w}}\left(\frac{1}{\lambda^{2}}+\frac{1}{\sigma^{2}}\right) d x \wedge d \bar{w} .
\end{aligned}
$$

The Bianchi identity and gauge field equations imply that $\lambda, \sigma$ satisfy

$$
\frac{1}{4} \partial_{x}^{2}\left(\frac{1}{\lambda^{2}} \pm \frac{1}{\sigma^{2}}\right)-\partial_{w} \partial_{\bar{w}}\left(\frac{1}{\lambda^{2}} \pm \frac{1}{\sigma^{2}}\right)=0
$$

In order to show that this solution satisfies the requirements to admit a (non-chiral) Majorana spinor, we first note that the condition (3.107) implies that there exist real functions $\mathcal{G}_{ \pm}$of the co-ordinates $x, w, \bar{w}$ which satisfy

$$
2 \partial_{x} \mathcal{G}_{ \pm}-4 \partial_{w} \mathcal{G}_{ \pm}=\frac{i}{2} \partial_{w}\left(\frac{1}{\lambda^{2}} \mp \frac{1}{\sigma^{2}}\right)+\frac{i}{4} \partial_{x}\left(\frac{1}{\lambda^{2}} \mp \frac{1}{\sigma^{2}}\right)
$$

The integrability conditions which are sufficient to imply local existence of such functions are equivalent to (3.107). We shall use the functions $\mathcal{G}_{ \pm}$to define the basis $W, \theta, \tau, V$ as follows:

$$
\begin{aligned}
W & =-4 d x-2 d w-2 d \bar{w} \\
\theta & =\frac{1}{2}\left(\lambda^{2}+\sigma^{2}\right)(d t+\phi)+\frac{i}{4}\left(\frac{1}{\lambda^{2}}-\frac{1}{\sigma^{2}}\right)(d w-d \bar{w})+\mathcal{G}_{+}(-4 d x-2 d w-2 d \bar{w}) \\
\tau & =-\frac{1}{2}\left(\lambda^{2}-\sigma^{2}\right)(d t+\phi)+\frac{i}{4}\left(\frac{1}{\lambda^{2}}+\frac{1}{\sigma^{2}}\right)(d w-d \bar{w})+\mathcal{G}_{-}(-4 d x-2 d w-2 d \bar{w})
\end{aligned}
$$

and

$$
\begin{aligned}
V= & \frac{1}{2}\left(-\mathcal{G}_{+}^{2}+\mathcal{G}_{-}^{2}\right)(-4 d x-2 d w-2 d \bar{w})-\frac{1}{8 \lambda^{2} \sigma^{2}}\left(d x-\frac{1}{2}(d w+d \bar{w})\right) \\
& +\mathcal{G}_{+}\left(-\frac{1}{2}\left(\lambda^{2}+\sigma^{2}\right)(d t+\phi)-\frac{i}{4}\left(\frac{1}{\lambda^{2}}-\frac{1}{\sigma^{2}}\right)(d w-d \bar{w})\right) \\
& +\mathcal{G}_{-}\left(-\frac{1}{2}\left(\lambda^{2}-\sigma^{2}\right)(d t+\phi)+\frac{i}{4}\left(\frac{1}{\lambda^{2}}+\frac{1}{\sigma^{2}}\right)(d w-d \bar{w})\right) .
\end{aligned}
$$

The metric given with respect to this basis in (3.44) then corresponds to the neutral IWP metric (3.103). $W$ is closed, and both $W$ and $\theta$ are co-closed. Furthermore, the gauge field strength satisfies

$$
F=-d \theta, \quad \star F=d \tau .
$$

Furthermore, all the remaining geometric conditions in (3.66) and (3.67) hold, as well as the Einstein equations.

We remark that the neutral IWP metric is in fact a $N=2$ Majorana solution. To see this, it is straightforward to show that the neutral IWP solution satisfies the Killing 
spinor equation (2.1), with a Dirac spinor $\eta=\lambda .1+\sigma e_{1}$. From such a Dirac spinor, one can construct two linearly independent non-chiral Majorana spinors

$$
\epsilon_{1}=\eta+C * \eta, \quad \epsilon_{2}=i(\eta-C * \eta)
$$

and $\epsilon_{1}$ (or $\left.\epsilon_{2}\right)$ is related to the spinor $1+e_{1}+e_{2}+e_{12}$ via an appropriately chosen $\operatorname{Spin}(2,2)$ gauge transformation.

\section{An alternative Killing spinor equation}

Instead of the Killing spinor equation (2.1), one can consider the following alternative Killing spinor equation

$$
D_{\mu} \epsilon=\nabla_{\mu} \epsilon-\frac{i}{4} \not F \Gamma_{\mu} \epsilon=0
$$

In this case, note that if $\epsilon$ satisfies (4.1) then so does $C * \gamma_{5} \epsilon$. Hence it is sufficient to consider spinors $\epsilon$ which satisfy $C * \gamma_{5} \epsilon=\epsilon$. A basis of such spinors over $\mathbb{R}$ is given by $\left\{\phi_{1}, \phi_{2}, \phi_{3}, \phi_{4}\right\}$ where

$$
\phi_{1}=\eta_{1}, \quad \phi_{2}=\eta_{2}, \quad \phi_{3}=-i \eta_{4}, \quad \phi_{4}=i \eta_{3} .
$$

The action of $\sigma_{1}, \sigma_{2}, i \sigma_{3}$ associated with (real) $\operatorname{Spin}(2,2)$ gauge transformations acting on $\operatorname{Span}_{\mathbb{R}}\left(\left\{\phi_{1}, \phi_{2}\right\}\right)$ is identical to the $\operatorname{SL}(2, \mathbb{R})$ generated by $T_{1}, T_{2}, T_{3}$ in (2.4).

The independent action of $\sigma_{1}, \sigma_{2}, i \sigma_{3}$ associated with (real) $\operatorname{Spin}(2,2)$ gauge transformations acting on $\operatorname{Span}_{\mathbb{R}}\left(\left\{\phi_{3}, \phi_{4}\right\}\right)$ is given by

$$
\hat{T}_{1}=-T_{1}, \quad \hat{T}_{2}=-T_{2}, \quad \hat{T}_{3}=T_{3} .
$$

So in this case there are three possible canonical spinors corresponding to

$$
\epsilon=\phi_{1}, \quad \text { or } \quad \epsilon=\phi_{3}, \quad \text { or } \quad \epsilon=\phi_{1}+\phi_{3}
$$

however $\phi_{1}$ and $\phi_{3}$ are also related by the $\operatorname{Pin}(2,2)$ transformation generated by $\gamma_{3}$, followed by rescaling with $i$, so there are two canonical spinors given by

$$
\epsilon=1+e_{12}, \quad \text { or } \quad \epsilon=1+e_{12}+e_{1}-e_{2} \text {. }
$$

The analysis of the case $\epsilon=1+e_{12}$ produces exactly the same conditions as for the $\operatorname{KSE}(2.1)$, i.e. it is a geometry of the type found in [28]. It remains to analyse the conditions obtained from (4.1) in the case $\epsilon=1+e_{12}+e_{1}-e_{2}$. However, in this case, it is straightforward to prove that the resulting linear system is identical to that given in (3.19) under the change of holomorphic basis indices $1 \leftrightarrow 2$ and $\overline{1} \leftrightarrow \overline{2}$.

Hence the supersymmetric solutions of (4.1) are in 1-1 correspondence with the supersymmetric solutions of (2.1) considered previously. 


\section{Conclusions}

In this paper we have classified the supersymmetric solutions of minimal ungauged 4dimensional supergravity in neutral signature. We find two classes of solutions with Majorana Killing spinors:

(i) The Killing spinor is chiral, and is parallel with respect to the Levi-Civita connection. The gauge field strength is self-dual, and the geometry admits a null-Kähler structure.

(ii) The Killing spinor is not chiral. These geometries admit a rotation-free null geodesic congruence $W$ which is constructed from a vector spinor bilinear, and with respect to which a parallel frame exists. Certain other geometric conditions given in (3.66), (3.67) must also hold.

We have also considered a number of examples in these two classes. The geometries in class (i) are a sub-case of those considered in [28], and their geometric structure is relatively straightforward to interpret, as all spinor bilinears must be parallel with respect to the Levi-Civita connection.

In contrast, for the class $(i i)$ solutions, the 2-form bilinear $\chi$ does not define a null almost-complex structure, although

$$
\omega^{ \pm}=W \wedge(\theta \pm \tau)
$$

which correspond to the self-dual/anti-self-dual parts of $\chi$, do define null-almost complex structures. However, the geometric conditions (3.66) and (3.67) do not appear to be sufficient to imply that either of $\omega^{+}$or $\omega^{-}$is integrable.

We remark that, for our general classification, we have concentrated solely on solutions preserving the minimal $N=1$ supersymmetry; though for some of the explicit examples we have considered $N=2$ solutions. In particular, if $\epsilon$ and $C * \epsilon$ are not proportional over $\mathbb{C}$, then $\eta_{1}=\epsilon+C * \epsilon$ and $\eta_{2}=i(\epsilon-C * \epsilon)$ are linearly independent (over $\mathbb{R}$ ) Majorana Killing spinors, i.e. the solution is really a $N=2$ solution. Hence, to understand solutions of this theory preserving the minimal $N=1$ supersymmetry, it is necessary and sufficient to consider the specific case of Majorana Killing spinors. We leave the classification of the $N=2$ solutions to future work.

Finally, we note that the issue of supersymmetry counting and Majorana Killing spinors is also of relevance in connection with the work of [31], in which solutions of the minimal gauged supergravity in neutral signature, and with nonzero cosmological constant $\Lambda$, were classified. The classification was undertaken for solutions with $\Lambda<0$ and $\Lambda>0$ separately. For the case $\Lambda<0$, the supercovariant derivative does not commute with the charge conjugation operator, and so the theory does not admit Majorana Killing spinors. However, for $\Lambda>0$, the supercovariant derivative does commute with charge conjugation, and Majorana Killing spinors should exist. Furthermore, one might expect (some of) the geometries found in our work to arise as appropriately tuned limits of $\Lambda>0$ solutions in 
from [31]. However, this is not the case, ${ }^{1}$ and the complete geometric interpretation of the class (ii) solutions remains elusive. It would be of interest to investigate these solutions further.

\section{Acknowledgments}

JG is supported by the STFC Consolidated Grant ST/L000490/1. The work of WS is supported in part by the National Science Foundation under grant number PHY-1620505. The authors would like to thank Maciej Dunajski for useful conversations.

\section{A Conventions}

We begin with a split signature (pseudo)-holomorphic basis, i.e. a basis

$$
\mathbf{e}^{1}, \quad \mathbf{e}^{2}, \quad \mathbf{e}^{\overline{1}}=\left(\mathbf{e}^{1}\right)^{*}, \quad \mathbf{e}^{\overline{2}}=\left(\mathbf{e}^{2}\right)^{*}
$$

with respect to which the metric is

$$
d s^{2}=2 \mathbf{e}^{1} \mathbf{e}^{\overline{1}}-2 \mathbf{e}^{2} \mathbf{e}^{\overline{2}} .
$$

With respect to this metric we define

$$
\Gamma_{1}=\sqrt{2} i_{e_{1}}, \quad \Gamma_{2}=\sqrt{2} i i_{e_{2}}, \quad \Gamma_{\overline{1}}=\sqrt{2} e_{1} \wedge, \quad \Gamma_{\overline{2}}=\sqrt{2} i e_{2} \wedge .
$$

The gamma matrices act either on the space of Dirac spinors, which consists of the complexified span of $\left\{1, e_{1}, e_{2}, e_{12}=e_{1} \wedge e_{2}\right\}$, or the subspace of Majorana spinors within the space of Dirac spinors. The canonical orbit elements differ depending on whether the spinors are Dirac or Majorana.

We will find it useful to also work with a real spacetime basis $\left\{\hat{\mathbf{e}}^{1}, \hat{\mathbf{e}}^{2}, \hat{\mathbf{e}}^{3}, \hat{\mathbf{e}}^{4}\right\}$ with respect to which the metric is

$$
d s^{2}=\left(\hat{\mathbf{e}}^{1}\right)^{2}+\left(\hat{\mathbf{e}}^{2}\right)^{2}-\left(\hat{\mathbf{e}}^{3}\right)^{2}-\left(\hat{\mathbf{e}}^{4}\right)^{2}
$$

and take

$$
\gamma_{1}=\frac{1}{\sqrt{2}}\left(\Gamma_{1}+\Gamma_{\overline{1}}\right), \quad \gamma_{2}=\frac{i}{\sqrt{2}}\left(\Gamma_{1}-\Gamma_{\overline{1}}\right)
$$

and

$$
\gamma_{3}=\frac{1}{\sqrt{2}}\left(\Gamma_{2}+\Gamma_{\overline{2}}\right), \quad \gamma_{4}=\frac{i}{\sqrt{2}}\left(\Gamma_{2}-\Gamma_{\overline{2}}\right)
$$

\footnotetext{
${ }^{1}$ In particular, for a non-chiral and Majorana Killing spinor, we have shown that one vector spinor bilinear vanishes, whereas the other one - which corresponds to $W$ - is nonzero. Moreover, all scalar spinor bilinears vanish. By comparison with [31], such solutions appear to be excluded via an algebraic analysis presented in appendix E.2 of that work; their 2-form $\Phi$ corresponds to the spinor 2-form bilinear $\chi$. However, we note that there is a mis-identification of the orbits of the space of 2 -forms, with respect to the action of $\mathrm{SO}(2,2)$, as given in equation (E.4) of [31]. Hence, a class of $N=1$ solutions with Majorana non-chiral Killing spinors has inadvertently been excluded from the classification of [31].
} 
With respect to the basis $\left\{1, e_{12}, e_{1}, e_{2}\right\}$, the gamma matrices $\gamma_{\mu}, \mu=1,2,3,4$ act as

$$
\begin{aligned}
\gamma_{1} & =\left(\begin{array}{ll}
0 & \mathbb{I} \\
\mathbb{I} & 0
\end{array}\right), & \gamma_{2} & =\left(\begin{array}{cc}
0 & i \sigma_{3} \\
-i \sigma_{3} & 0
\end{array}\right), \\
\gamma_{3} & =\left(\begin{array}{cc}
0 & -\sigma_{2} \\
\sigma_{2} & 0
\end{array}\right), & \gamma_{4} & =\left(\begin{array}{cc}
0 & -\sigma_{1} \\
\sigma_{1} & 0
\end{array}\right)
\end{aligned}
$$

with $^{2}$

$$
\sigma_{1}=\left(\begin{array}{cc}
0 & 1 \\
1 & 0
\end{array}\right), \quad \sigma_{2}=\left(\begin{array}{cc}
0 & -i \\
i & 0
\end{array}\right), \quad \sigma_{3}=\left(\begin{array}{cc}
1 & 0 \\
0 & -1
\end{array}\right)
$$

Majorana spinors $\epsilon$ satisfy the condition $\epsilon=C * \epsilon$, where

$$
C=\left(\begin{array}{cc}
\sigma_{1} & 0 \\
0 & \sigma_{1}
\end{array}\right)
$$

satisfies

$$
\left[C *, \gamma_{\mu}\right]=0
$$

with

$$
C * 1=e_{12}, \quad C * e_{12}=1, \quad C * e_{1}=e_{2}, \quad C * e_{2}=e_{1} .
$$

It is useful to note that

$$
\begin{aligned}
\gamma_{12} & =\left(\begin{array}{cc}
-i \sigma_{3} & 0 \\
0 & i \sigma_{3}
\end{array}\right), & \gamma_{34} & =\left(\begin{array}{cc}
i \sigma_{3} & 0 \\
0 & i \sigma_{3}
\end{array}\right) \\
\gamma_{13} & =\left(\begin{array}{cc}
\sigma_{2} & 0 \\
0 & -\sigma_{2}
\end{array}\right), & \gamma_{24} & =\left(\begin{array}{cc}
-\sigma_{2} & 0 \\
0 & -\sigma_{2}
\end{array}\right) \\
\gamma_{14} & =\left(\begin{array}{cc}
\sigma_{1} & 0 \\
0 & -\sigma_{1}
\end{array}\right), & \gamma_{23} & =\left(\begin{array}{cc}
\sigma_{1} & 0 \\
0 & \sigma_{1}
\end{array}\right) .
\end{aligned}
$$

The action of the (real) Spin(2,2) transformations generated by $\gamma_{12} \pm \gamma_{34}, \gamma_{13} \pm \gamma_{24}$ and $\gamma_{14} \pm \gamma_{23}$ decomposes to (independent) actions of $\left\{\sigma_{1}, \sigma_{2}, i \sigma_{3}\right\}$ on the spans of basis elements $\left\{1, e_{12}\right\}$ and $\left\{e_{1}, e_{2}\right\}$.

A $\operatorname{Spin}(2,2)$ invariant inner product $\mathcal{B}$ is given by

$$
\mathcal{B}(\epsilon, \eta)=\langle B \epsilon, \eta\rangle
$$

where

$$
B=\left(\begin{array}{cc}
\sigma_{3} & 0 \\
0 & \sigma_{3}
\end{array}\right)
$$

\footnotetext{
${ }^{2} \sigma_{i} \sigma_{j}=\delta_{i j} \mathbb{I}+i \epsilon_{i j k} \sigma_{k}$.
} 
satisfies

$$
B .1=1, \quad B . e_{12}=-e_{12}, \quad B . e_{1}=e_{1}, \quad B \cdot e_{2}=-e_{2} .
$$

This inner product satisfies

$$
\mathcal{B}\left(\epsilon, \gamma_{\mu} \eta\right)=\mathcal{B}\left(\gamma_{\mu} \epsilon, \eta\right)
$$

and also

$$
\mathcal{B}\left(\epsilon, \gamma_{\mu \nu} \eta\right)=-\mathcal{B}\left(\gamma_{\mu \nu} \epsilon, \eta\right)
$$

Furthermore, if both $\epsilon, \eta$ are Majorana, then

$$
\mathcal{B}(\epsilon, \eta)=-\mathcal{B}(\eta, \epsilon)
$$

Also, note that

$$
\Gamma_{5} \equiv \gamma_{1234}=-\Gamma_{1 \overline{1} 2 \overline{2}}=\left(\begin{array}{cc}
\mathbb{I} & 0 \\
0 & -\mathbb{I}
\end{array}\right) .
$$

If we set $\epsilon_{1 \overline{1} 2 \overline{2}}=1$ with respect to the complex frame (A.2), this implies that $\epsilon_{1234}=-1$ with respect to the real frame (A.4).

\section{B Analysis of condition (3.40)}

In order to analyse the condition (3.40), we introduce a local real basis $\left\{\mathbf{E}^{i}: i=1,2,3,4\right\}$ such that

$$
d s^{2}=2 \mathbf{E}^{1} \mathbf{E}^{2}+2 \mathbf{E}^{3} \mathbf{E}^{4}
$$

with $W=\mathbf{E}^{1}$. Then in this basis,

$$
\theta=\alpha \mathbf{E}^{1}+\mathbf{E}^{3}+\frac{1}{2} \mathbf{E}^{4} .
$$

We take the volume form to be dvol $=\mathbf{E}^{1} \wedge \mathbf{E}^{2} \wedge \mathbf{E}^{3} \wedge \mathbf{E}^{4}$. In this basis the conditions (3.37) and (3.38) are equivalent to

$$
\nabla_{1} \theta_{2}+\nabla_{2} \theta_{1}+\nabla_{3} \theta_{4}+\nabla_{4} \theta_{3}=0
$$

and

$$
\nabla_{2} \theta_{\mu}=0
$$

Then (3.40) implies that the remaining independent condition obtained from (3.40) is

$$
\alpha\left(\nabla_{4} \theta_{3}-\nabla_{3} \theta_{4}\right)-\frac{1}{2} \nabla_{1} \theta_{3}+\nabla_{3} \theta_{1}+\nabla_{1} \theta_{4}-2 \nabla_{4} \theta_{1}=0 .
$$


On making use of this condition, one finds that

$$
(\theta \wedge d \theta)_{134}=-\nabla_{4} \theta_{1}+\frac{1}{2} \nabla_{3} \theta_{1}
$$

and hence

$$
\star(\theta \wedge d \theta)_{1}=-(\theta \wedge d \theta)_{134}=\nabla_{4} \theta_{1}-\frac{1}{2} \nabla_{3} \theta_{1}=\left(\nabla_{\tau} \theta\right)_{1}
$$

where $\tau=\mathbf{E}^{3}-\frac{1}{2} \mathbf{E}^{4}$. The remaining components of (3.41) follow automatically. Furthermore, we remark that if $\tau$ satisfies $\tau^{2}=-1$, and is orthogonal to $W, \theta$ with respect to the basis (B.1), then the most general expression for $\tau$ is given by

$$
\tau=\beta \mathbf{E}^{1} \pm\left(\mathbf{E}^{3}-\frac{1}{2} \mathbf{E}^{4}\right)
$$

however the term parallel to $\mathbf{E}^{1}$, whose coefficient $\beta$ is not determined uniquely, gives no contribution to $\nabla_{\tau} \theta$ appearing in (3.41), as a consequence of (3.38).

\section{Analysis of condition (3.39)}

On making use of the conditions on $\nabla \theta$ given in (3.37), (3.38), and (3.41), the condition (3.39) can be rewritten as $d W=0$ together with

$$
\nabla_{W} W=0
$$

and

$$
\begin{aligned}
& \nabla_{1} W_{1}=2 \alpha \nabla_{3} \theta_{4}+\nabla_{1} \theta_{3}-2 \nabla_{3} \theta_{1} \\
& \nabla_{1} W_{3}=2 \nabla_{3} \theta_{4} \\
& \nabla_{1} W_{4}=\nabla_{4} \theta_{3} \\
& \nabla_{3} W_{3}=-2 \nabla_{3} \theta_{2} \\
& \nabla_{3} W_{4}=0 \\
& \nabla_{4} W_{4}=-\nabla_{4} \theta_{2} .
\end{aligned}
$$

Note that (C.2) is equivalent to

$$
\nabla_{\tau} W=\star(W \wedge d \theta)
$$

and

$$
\nabla_{1} W_{1}=-2\left(i_{\theta} d \theta\right)_{1}
$$

The condition (C.4) can be rewritten, on taking

$$
\tau=\mathbf{E}^{3}-\frac{1}{2} \mathbf{E}^{4}
$$


and

$$
V=\mathbf{E}^{2}-\alpha\left(\mathbf{E}^{3}+\frac{1}{2} \mathbf{E}^{4}\right)-\frac{1}{2} \alpha^{2} \mathbf{E}^{1} .
$$

Then (C.4) can be rewritten as

$$
\nabla_{V} W=\star(\tau \wedge d \theta)-i_{\theta} d \theta .
$$

The relationship between the frame $\left\{\mathbf{E}^{1}, \mathbf{E}^{2}, \mathbf{E}^{3}, \mathbf{E}^{4}\right\}$ and $\{V, W, \theta, \tau\}$ is

$$
\begin{aligned}
W & =\mathbf{E}^{1} \\
V & =\mathbf{E}^{2}-\alpha\left(\mathbf{E}^{3}+\frac{1}{2} \mathbf{E}^{4}\right)-\frac{1}{2} \alpha^{2} W \\
\tau & =\mathbf{E}^{3}-\frac{1}{2} \mathbf{E}^{4} \\
\theta & =\alpha \mathbf{E}^{1}+\mathbf{E}^{3}+\frac{1}{2} \mathbf{E}^{4}
\end{aligned}
$$

and hence the metric is

$$
d s^{2}=2 V W+\theta^{2}-\tau^{2}
$$

with volume form dvol $=W \wedge V \wedge \tau \wedge \theta$.

Open Access. This article is distributed under the terms of the Creative Commons Attribution License (CC-BY 4.0), which permits any use, distribution and reproduction in any medium, provided the original author(s) and source are credited.

\section{References}

[1] K.p. Tod, All Metrics Admitting Supercovariantly Constant Spinors, Phys. Lett. B 121 (1983) 241 [INSPIRE].

[2] K.P. Tod, More on supercovariantly constant spinors, Class. Quant. Grav. 12 (1995) 1801 [INSPIRE].

[3] J.P. Gauntlett, J.B. Gutowski, C.M. Hull, S. Pakis and H.S. Reall, All supersymmetric solutions of minimal supergravity in five-dimensions, Class. Quant. Grav. 20 (2003) 4587 [hep-th/0209114] [INSPIRE].

[4] M.M. Caldarelli and D. Klemm, All supersymmetric solutions of $N=2, D=4$ gauged supergravity, JHEP 09 (2003) 019 [hep-th/0307022] [INSPIRE].

[5] S.L. Cacciatori, D. Klemm, D.S. Mansi and E. Zorzan, All timelike supersymmetric solutions of $N=2, D=4$ gauged supergravity coupled to abelian vector multiplets, JHEP 05 (2008) 097 [arXiv: 0804.0009] [INSPIRE].

[6] D. Klemm and E. Zorzan, All null supersymmetric backgrounds of $N=2, D=4$ gauged supergravity coupled to abelian vector multiplets, Class. Quant. Grav. 26 (2009) 145018 [arXiv: 0902.4186] [INSPIRE].

[7] J.P. Gauntlett and S. Pakis, The Geometry of D = 11 Killing spinors, JHEP 04 (2003) 039 [hep-th/0212008] [INSPIRE]. 
[8] J.P. Gauntlett, J.B. Gutowski and S. Pakis, The Geometry of D =11 null Killing spinors, JHEP 12 (2003) 049 [hep-th/0311112] [INSPIRE].

[9] P. Meessen, T. Ortín and S. Vaula, All the timelike supersymmetric solutions of all ungauged $d=4$ supergravities, JHEP 11 (2010) 072 [arXiv:1006.0239] [INSPIRE].

[10] J. Bellorín and T. Ortín, Characterization of all the supersymmetric solutions of gauged $N=1, d=5$ supergravity, JHEP 08 (2007) 096 [arXiv: 0705.2567] [INSPIRE].

[11] M. Akyol and G. Papadopoulos, Spinorial geometry and Killing spinor equations of $6-D$ supergravity, Class. Quant. Grav. 28 (2011) 105001 [arXiv:1010.2632] [InSPIRE].

[12] M. Akyol and G. Papadopoulos, $(1,0)$ superconformal theories in six dimensions and Killing spinor equations, JHEP 07 (2012) 070 [arXiv: 1204.2167] [INSPIRE].

[13] M. Akyol and G. Papadopoulos, Brane solitons of $(1,0)$ superconformal theories in six dimensions with hyper-multiplets, Class. Quant. Grav. 31 (2014) 065012 [arXiv:1307.1041] [INSPIRE].

[14] P.A. Cano and T. Ortín, The structure of all the supersymmetric solutions of ungauged $\mathcal{N}=(1,0), d=6$ supergravity, Class. Quant. Grav. 36 (2019) 125007 [arXiv:1804.04945] [INSPIRE].

[15] H. Het Lam and S. Vandoren, BPS solutions of six-dimensional $(1,0)$ supergravity coupled to tensor multiplets, JHEP 06 (2018) 021 [arXiv:1804.04681] [INSPIRE].

[16] H. Blaine Lawson and Marie-Louise Michelsohn, Spin geometry, Princeton University Press, Princeton U.S.A. (1989).

[17] F. Reese Harvey, Spinors and calibrations, Academic Press New York U.S.A. (1990).

[18] J. Gillard, U. Gran and G. Papadopoulos, The Spinorial geometry of supersymmetric backgrounds, Class. Quant. Grav. 22 (2005) 1033 [hep-th/0410155] [INSPIRE].

[19] U. Gran, G. Papadopoulos and D. Roest, Systematics of M-theory spinorial geometry, Class. Quant. Grav. 22 (2005) 2701 [hep-th/0503046] [INSPIRE].

[20] U. Gran, J. Gutowski and G. Papadopoulos, The Spinorial geometry of supersymmetric IIB backgrounds, Class. Quant. Grav. 22 (2005) 2453 [hep-th/0501177] [INSPIRE].

[21] U. Gran, J. Gutowski and G. Papadopoulos, The $G_{2}$ spinorial geometry of supersymmetric IIB backgrounds, Class. Quant. Grav. 23 (2006) 143 [hep-th/0505074] [INSPIRE].

[22] U. Gran, P. Lohrmann and G. Papadopoulos, The Spinorial geometry of supersymmetric heterotic string backgrounds, JHEP 02 (2006) 063 [hep-th/0510176] [INSPIRE].

[23] U. Gran, J. Gutowski and G. Papadopoulos, Geometry of all supersymmetric four-dimensional $N=1$ supergravity backgrounds, JHEP 06 (2008) 102 [arXiv:0802.1779] [INSPIRE].

[24] U. Gran, J. Gutowski and G. Papadopoulos, Classification, geometry and applications of supersymmetric backgrounds, Phys. Rept. 794 (2019) 1 [arXiv: 1808.07879] [INSPIRE].

[25] M. Dunajski, J. Gutowski, W. Sabra and P. Tod, Cosmological Einstein-Maxwell Instantons and Euclidean Supersymmetry: Anti-Self-Dual Solutions, Class. Quant. Grav. 28 (2011) 025007 [arXiv: 1006.5149 ] [INSPIRE].

[26] J.B. Gutowski and W.A. Sabra, Gravitational Instantons and Euclidean Supersymmetry, Phys. Lett. B 693 (2010) 498 [arXiv:1007.2421] [INSPIRE]. 
[27] M. Dunajski, J.B. Gutowski, W.A. Sabra and P. Tod, Cosmological Einstein-Maxwell Instantons and Euclidean Supersymmetry: Beyond Self-Duality, JHEP 03 (2011) 131 [arXiv: 1012.1326] [INSPIRE].

[28] R.L. Bryant, Pseudo-Riemannian metrics with parallel spinor fields and vanishing Ricci tensor, Sémin. Congr. Soc. Math. France 4 (2000) 53.

[29] M. Dunajski, Antiselfdual four manifolds with a parallel real spinor, Proc. Roy. Soc. Lond. A 458 (2002) 1205 [math/0102225] [INSPIRE].

[30] M. Dunajski, Null Kahler structures, symmetries and integrability, Topics in Mathematical Physics, General Relativity and Cosmology in Honor of Jerzy Plebanski. Proceedings of 2002 International Conference, Mexico City Mexico (2002), H. García-Compeán, B. Mielnik, M. Montesinos and J. Przanowski eds., World Scientific, Singapore (2006).

[31] D. Klemm and M. Nozawa, Geometry of Killing spinors in neutral signature, Class. Quant. Grav. 32 (2015) 185012 [arXiv: 1504.02710] [INSPIRE].

[32] C.M. Hull, Duality and the signature of space-time, JHEP 11 (1998) 017 [hep-th/9807127] [INSPIRE].

[33] W.A. Sabra, Special geometry and space-time signature, Phys. Lett. B 773 (2017) 191 [arXiv: 1706.05162] [INSPIRE].

[34] J.W. Barrett, G.W. Gibbons, M.J. Perry, C.N. Pope and P. Ruback, Kleinian geometry and the $N=2$ superstring, Int. J. Mod. Phys. A 9 (1994) 1457 [hep-th/9302073] [INSPIRE]. 